\title{
Odor and the Effects of Schema Activation on Recognition Memory
}

\author{
Kelly Appino, Claire Svec, Brandon Tankard, Amy A. Overman \\ Department of Psychology \\ Elon University \\ Elon, North Carolina 27244, USA \\ Joseph D. W. Stephens \\ Department of Psychology \\ North Carolina A \& T State University \\ Greensboro, North Carolina 27411 USA
}

Received: June 11, 2010 Accepted: November 19, 2010

\begin{abstract}
This study investigated whether schema-consistent odors affect recognition memory. Forty-two undergraduate students read a story about baking in the presence of an odor that was either baking-related (schema-consistent) or not (schema-inconsistent). Further, the story contained information that was both inconsistent, and consistent, with baking. Participants were then tested on recognition of information from the story. It was predicted that participants who smelled the schema-consistent odor would be more likely to falsely recognize new (i.e., not in the story) schema-consistent information than those who smelled the schema-inconsistent odor. The results indicated that all participants were more likely to falsely recognize new schema-consistent information than schema-inconsistent information. However, odor had no statistically significant effects on recognition.
\end{abstract}

\section{ODOR AND THE EFFECTS OF SCHEMA ACTIVATION ON RECOGNITION MEMORY}

Olfaction (sense of smell) is commonly thought to be very powerful in evoking vivid autobiographical memories, and this popular intuition has been supported by recent scientific research in cognitive psychology (e.g., [1]). However, the precise ways in which odors interact with memory are still not fully understood. For example, having an odor present during encoding and retrieval can improve memory for lists of words [2] and for familiar and unfamiliar items [3], and this effect can be increased by the distinctiveness of the odor [4]. On the other hand, some evidence suggests that certain odors (e.g., lavender) improve memory while others (e.g., jasmine) hinder memory performance [5].
The current study investigated whether odors can modulate the effects of schema activation on memory for details of a story. Schemas are hypothetical knowledge structures that may consist of information that is typically true of certain categories or situations in the world. As such, schemas help the mind to organize knowledge and expectations, thereby guiding how it perceives the world and serving as a basis for searching and reconstructing memory (e.g., [6]). For example, when entering a room that is labeled as an office, a person will have certain expectations of what is contained in that room and the activities that take place there. Consequently, this set of expectations can influence what one later remembers about the room [7, 8]. In particular, one may incorrectly remember something that was not in the room but is consistent with the schema (such as a stapler); one may also 
have better memory for items that are inconsistent with the schema because they violate expectations and become more noticeable (e.g., [7, 9]).

Very little prior research has addressed the question of whether schema effects on memory can be influenced by odor cues. One study of the effects of odor on memory for words [2] did find that odor had different effects depending on whether the words were related or unrelated to the odor. Specifically, odor increased recall of words that were unrelated to the odor but not words that were related to the odor, compared to a condition in which participants were instructed to imagine the odor, but did not actually smell it. It was suggested that the instruction to imagine the smell may have activated the concepts related to the smell strongly enough that the addition of the odor itself could not further increase the effect. This finding may relate to schema effects because it may be possible that once a schema is activated, the presence of odors that are consistent or inconsistent with the schema may not be able to change the schema's effect on memory. On the other hand, it is realistic to think that since odors serve as a good memory cue, they may boost the effects of schema activation when consistent with the schema and diminish the effects of schema activation when inconsistent with the schema.

The present experiment addressed these questions explicitly by presenting participants with a story designed to activate a schema of baking cookies. One group of participants read the story while smelling a sweet, baking-related scent and another group read the story while smelling a scent that was unrelated to baking. It was hypothesized that, if odors can influence schema effects on memory, the schemaconsistent odor would lead to increases in false recognition of schema-consistent details that were not present in the story, compared to when the schema-inconsistent odor was used.

\section{METHOD}

\section{a. Participants}

Forty-two male and female undergraduate students, between the ages of 18 and 22 (mean age $=18.58$ years), from Elon University volunteered to participate in our study. Participants received extra credit in their courses for participation in our study. Participants were pre-screened to exclude anyone with allergies or asthma. This research was approved by Elon University IRB prior to being conducted.

\section{b. Design}

This experiment was a 2 (odor type) $X 2$ (information type) between-subjects design. The type of odor had two levels (schema-consistent odor, schemainconsistent odor) and the type of information read had two levels (schemaconsistent information, schema-inconsistent information).

\section{c. Materials}

A baking story was presented in 12pt. Times New Roman font on a white sheet of paper. The story was titled Susie's Baking Adventure, and described a scenario in which the main character, Susie, baked cookies. The story had equal amounts of information that was consistent with baking cookies (e.g., adding sugar), and inconsistent with baking cookies (e.g., getting paprika out of the cabinet). Two different oil-based odors were used. Warm Vanilla Sugar, which was consistent with a cookie-baking schema as it smelled like a sweet, sugary dessert, and Dancing Waters, which was inconsistent with a cookie-baking schema as it had an ocean scent.

\section{d. Procedure}

Participants were randomly assigned to either a schema-consistent or schema-inconsistent odor group. The odor was prepared by dipping a standard-sized cotton ball in the oil and then placing it in a small plastic storage bag with a sealable closure. Twelve cotton balls were placed in each odor storage bag. The storage bag was then opened when a participant was reading the informed consent form. After the participant signed the informed consent form he/she was instructed to position their nose approximately 2 inches from the unsealed odor storage bag and to inhale the odor for 
30 seconds. The bag was then placed just above the sheet of paper, where it continued to emit odor while the participant read the story once. The bag was then closed and removed. Participants then were asked to silently count backwards from 3000 by twos for five minutes. This distractor task was incorporated so that participants would not rehearse the story and so that recency effects would be decreased. After, a recognition memory task was administered via PowerPoint on an overhead screen in the testing room. It contained twenty phrases presented for 10 seconds each: 5 items that were baking-related and were also in the story (schema-consistent targets), 5 items that were baking-related and were not in the story (schemaconsistent distractors), 5 items that were not baking-related and were in the story (schema-inconsistent targets), and 5 items that were not baking-related and were not in the story (schema-inconsistent distractors). Participants indicated whether they remembered the phrases from the story by circling 'yes' or 'no' on a sheet of paper. The answer sheet did not contain the phrases. It only contained 'yes' and 'no' choices. After the recognition task the participants were debriefed both orally and in writing.

\section{RESULTS}

For each participant, the proportions of "yes" responses to targets (hits) and distractors (false alarms) were computed for schema-consistent and schema-inconsistent items in the recognition memory task. These data are presented in Figure 1. The response proportions were examined in a 2 (response category: hits vs. false alarms) $\mathrm{X}$ 2 (information type: schema-consistent items vs. schema-inconsistent items) $\times 2$ (odor type: schema-consistent odor vs. schema-inconsistent odor) mixed-design ANOVA. A significant main effect of response category was found, $F(1,40)=$ $38.1, p<.001, \eta_{p}^{2}=.49$, reflecting a higher overall rate of hits than of false alarms. This indicates that participants recognized information from the story more than information that was not in the story and were not just answering "yes" to everything. No significant main effects were found for information type $F(1,40)=2.94, p=.09, \eta_{p}^{2}$
$=.07$, or for odor type, $F(1,40)=.09, p=$ .76, $\eta_{p}^{2}=.002$.

A significant interaction of response category $X$ information type was found, $F(1,40)=4.66, p=.037, \eta_{p}^{2}=.10$, reflecting a larger difference between hits and false alarms for schema-inconsistent information than for schema-consistent information. That is, participants were better able to distinguish between old and new items when those items were inconsistent with the baking schema. It can be seen from the figure that the primary difference in response patterns between the two information types was in the false alarms rate: participants were more likely to falsely remember new items that were consistent with the baking schema. This observation was confirmed by a paired-samples $t$-test on the false alarm rates for schema-consistent and schema-inconsistent information (collapsed across odor groups), $t(41)=3.42$, $p=.001, d=.60$.

There were no interactions of response category $X$ odor type, $F(1,40)=$ $.03, p=.87, \eta_{p}^{2}=.001$, information type $X$ odor type, $F(1,40)=.21, p=.65, \eta_{p}^{2}=.005$, or response category $X$ information type $X$ odor type, $F(1,40)=.01, p=.92, \eta_{p}^{2}<.001$. Thus, odor type did not influence schema effects on memory.

\section{DISCUSSION}

The current study examined schema effects on recognition memory, and whether these effects could be influenced by exposure to odors during memory encoding. Participants read a brief story about baking and were then tested on recognition memory for items that were either baking-related (schema-consistent information) or not baking-related (schema-inconsistent information). One group of participants smelled a baking-related odor (schemaconsistent odor) while reading the story, and another group of participants smelled a nonbaking-related odor (schema-inconsistent odor) while reading the story. It was hypothesized that the baking-related odor would more strongly activate baking-related concepts and enhance schema effects on memory in the recognition task.

The results indicated that activation of a baking schema did affect memory in the recognition task. Specifically, participants 

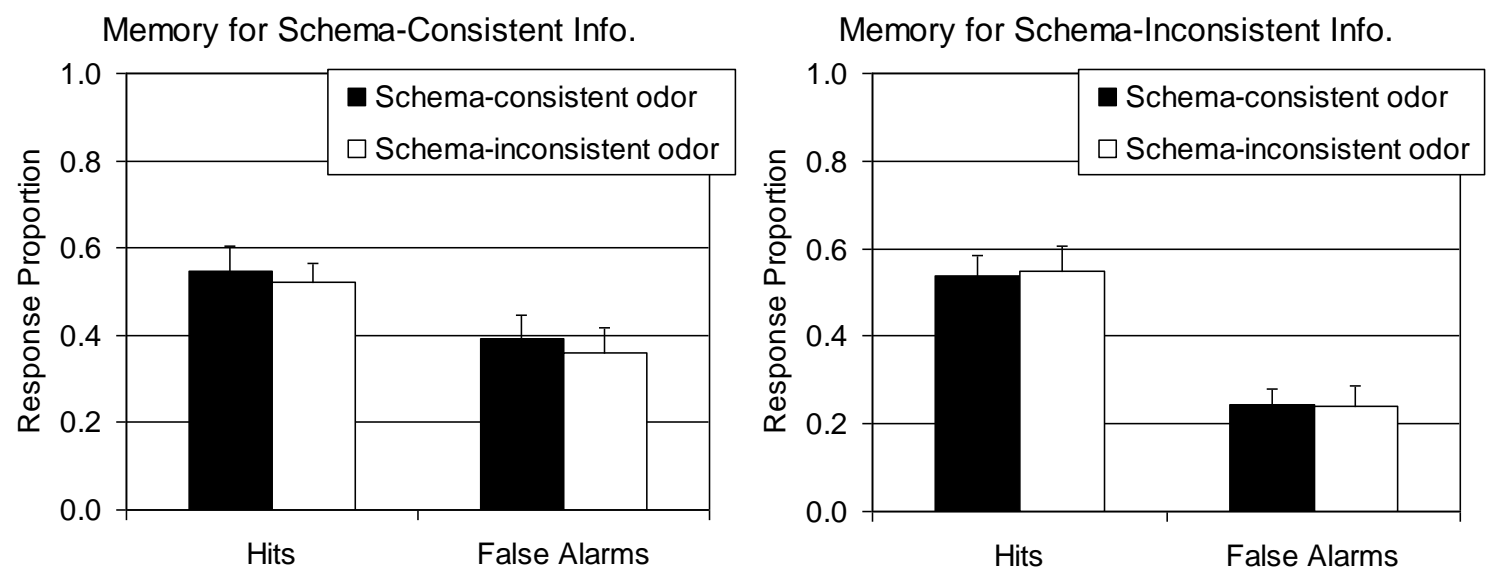

Figure 1. Proportion "yes" responses to items in the recognition memory task. Hits are "yes" responses to items that were contained in the narrative passage read by participants during the study phase; false alarms are "yes" responses to items that were not contained in the narrative passage. Items presented in the memory task were either consistent with the baking schema of the studied narrative (left panel) or inconsistent with the baking schema (right panel). During the study phase, one group of participants was exposed to an odor consistent with the baking schema (black bars) and the other group of participants was exposed to an odor not consistent with the baking schema (white bars). Error bars represent standard error of the mean.

were more likely to incorrectly remember baking-related items that were not in the story, compared to non-baking-related items that were not in the story. This finding replicates many previous studies of schema effects on memory, in which participants are more likely to falsely remember items that are consistent with the overall schema activated during encoding (e.g., [7, 09].

Regarding the influence of odor, a close inspection of the data displayed in Figure 1 indicates that participants who smelled the schema-consistent odor were slightly more likely to recognize schemaconsistent information compared to participants who smelled the schemainconsistent odor. This pattern is consistent with the hypothesis that schema-consistent odors may contribute to recognition of schema-consistent information. However, statistical analyses found no significant differences due to odor in the experiment. If odor does influence schema effects in memory, its effects were not reliable in this experiment. This finding is consistent with the results of Schab (1990), in which recall of odor-related words was the same when participants were instructed to think about and imagine an odor, compared to when they both thought about an odor and also smelled it at the same time. However, that study did not directly test schema activation effects and their relation to odor. The current study makes a unique contribution by extending the prior research and bringing together two areas of memory research (schema effects and odor) that had not previously been combined.

Further experiments may be able to detect odor effects (if they exist) by testing a larger number of participants and by testing each participant on a larger number of items in order to obtain more precise memory data. It may also have been the case that the baking schema was so strongly activated by the story in this experiment that the odors presented to participants were not able to increase or schema activation by very much. Another possibility is that the schema-related odor used here ("Warm Vanilla Sugar") was not as effective as possible in contributing to the baking schema. For example, it is a popular scent and may have been familiar to participants in non-baking contexts, or it may contain elements that take away from its similarity to the specific concepts associated with the baking schema activated by the story.

This study makes a contribution to the literature because previous research has 
not directly investigated the influence of odor on schema-based memory effects. This is an important area to study because the role of odor in memory is still poorly understood as is any potential relationship between odor and schema with regard to memory performance. Further experiments like this one will help to clarify to what extent these senses contribute to well-known memory phenomena such as the effects of schema activation.

\section{REFERENCES}

1. Chu, S., \& Downes, J. J. (2002). Proust nose best: Odors are better cues of autobiographical memory. Memory \& Cognition, 30(4), 511-518.

2. Schab, F. R. (1990). Odors and the remembrance of things past. Journal of Experimental Psychology: Learning, Memory, and Cognition, 16(4), 648-655.

3. Morrin, M., \& Ratneshwar, S. (2003). Does it make sense to use scents to enhance brand memory? Journal of Marketing Research, 40(1), 10-25.

4. Herz, R. S. (1997). The effects of cue distinctiveness on odor-based context- dependent memory. Memory \& Cognition, 25, 375-380.

5. Degel, J., \& Köster, E. P. (1999). Odors: Implicit memory and performance effects. Chemical Senses, 24(3), 317325.

6. Neuschatz, J. S., Lampinen, J. M., Preston, E. L., Hawkins, E. R., \& Toglia, M. P. (2002). The effect of memory schemata on memory and the phenomenological experience of naturalistic situations. Applied Cognitive Psychology, 16, 687-708.

7. Brewer, W. F., \& Treyens, J. C. (1981). Role of schemata in memory for places. Cognitive Psychology, 13(2), 207-230.

8. Kleider, H.M., Goldinger, S.D., \& Knuycky, L. (2008). Stereotypes influence false memories for imagined events. Psychology Press, 16(2), 97114.

9. Lampinen, J. M., Copeland, S.M., \& Neuschatz, J. S. (2001). Recollection of things schematic: Room schemas revisited. Journal of Experimental Psychology, 27, 1211-1222.

\section{ELON UNNIVERSITY $\bullet$ Elon, North Carolina}

Elon students work closely with distinguished faculty in an innovative liberal arts community that values global perspective and concern for the greater good. www.elon.edu

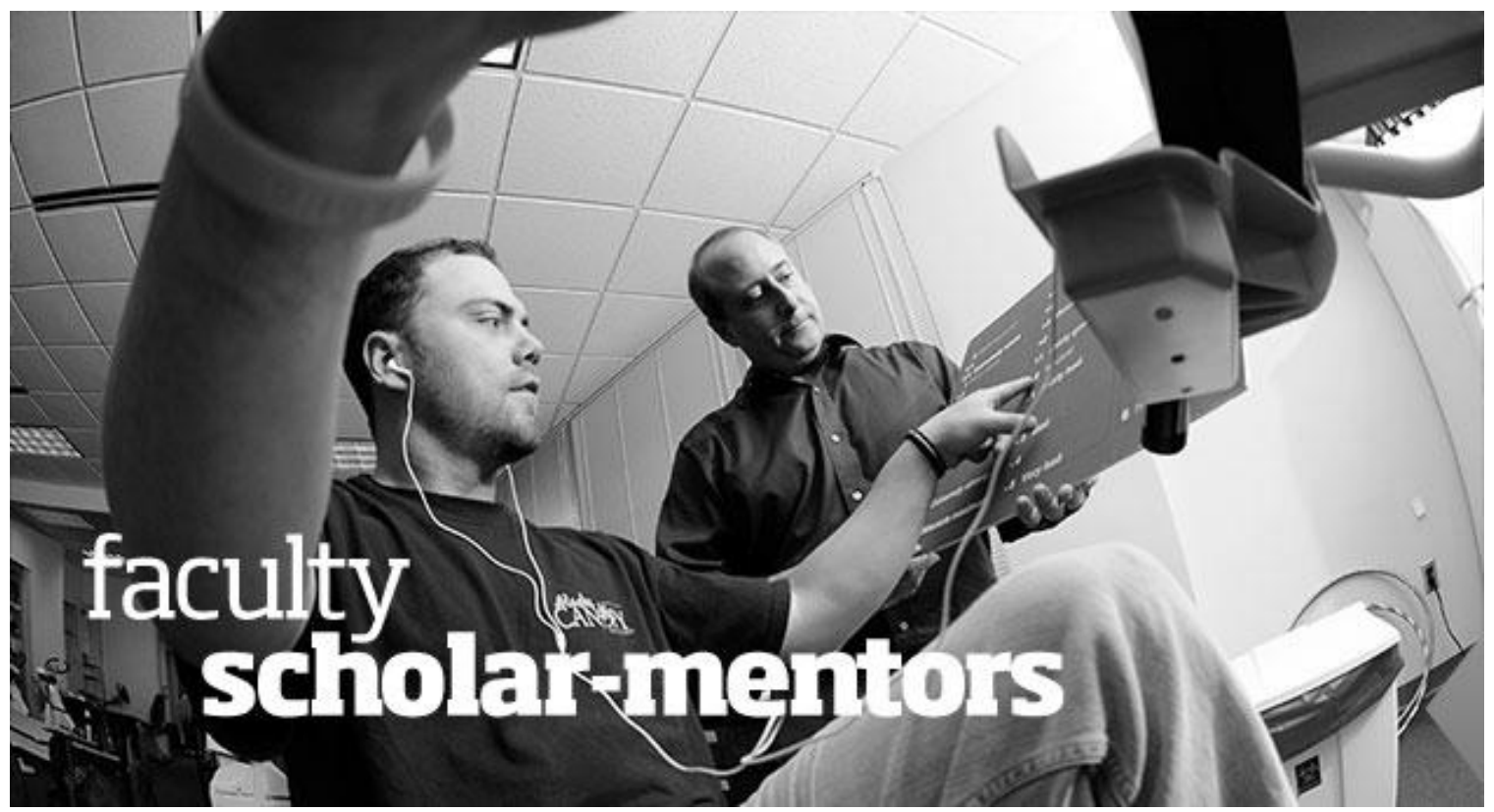




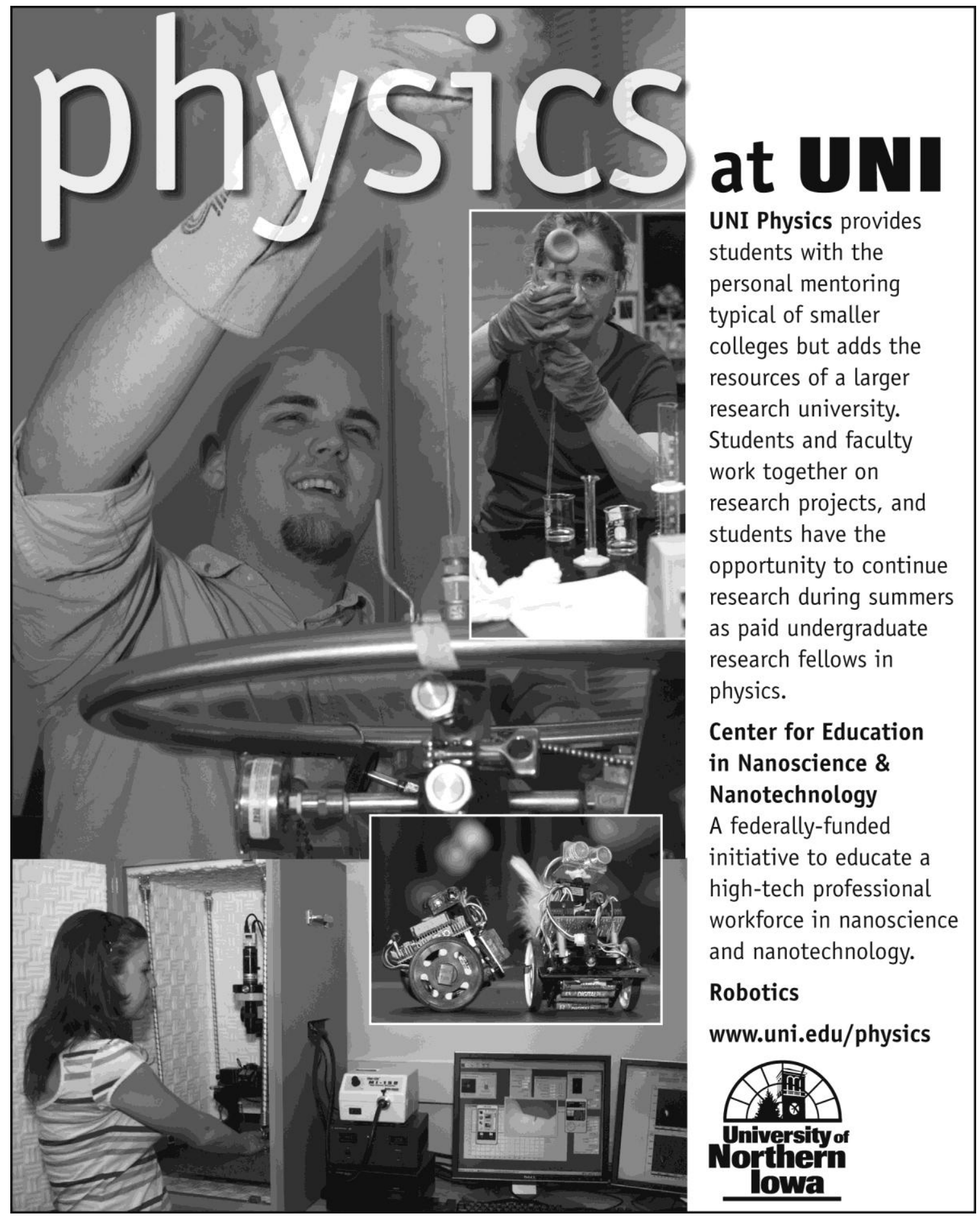

